\title{
Rinoplastía: resultados desde la perspectiva del paciente. Validación lingüística y psicométrica del Rhinoplasty Outcome Evaluation Instrument*
}

\author{
Drs. M. ELSA CALDERÓN G. ${ }^{1}$, PEDRO CUEVAS T. ${ }^{1}$, CRISTIAN ERAZO C. ${ }^{1}$, SUSANA BENÍTEZ S. ${ }^{1}$, \\ PATRICIO ANDRADES C. ${ }^{1}$, SERGIO SEPÚLVEDA P. ${ }^{1}$, Als. JOSÉ IGNACIO VERGARA O. ${ }^{2}$, \\ FELIPE SOTO V. ${ }^{2}$, ALMA CRUZ ${ }^{2}$, Dr. STEFAN DANILLA E. ${ }^{1}$
}

\footnotetext{
Departamento de Cirugía, Hospital Clínico de la Universidad de Chile.

2 Alumnos Facultad de Medicina Universidad de Chile.

Santiago, Chile.
}

\begin{abstract}
Validation of the spanish version of the Rhinoplasty Outcome Evaluation

Background: The Rhinoplasty Outcome Evaluation is a questionnaire that assesses, form the point of view of the patient, the functional and cosmetic outcome of rhinoplasty. It has six items for esthetic and functional domains, using Likert type questions with five alternatives. The scale ranges from 6 that is the worst outcome to 30, the best. Aim: To translate and validate the Rhinoplasty Outcome Evaluation, to be used in Chile. Material and Methods: The linguistic validation guidelines of the MAPI/TRUST Research Institute were used. The instrument was translated from English to Spanish, counter translated and applied to a pilot sample of five patients. The internal stability was assessed using Cronbach alpha. Results: The five female patients in whom the questionnaire was applied were aged $22 \pm 4$ years, had $15 \pm 2$ years of studies and their body mass index was $23 \pm 4 \mathrm{~kg} / \mathrm{m}^{2}$. Cronbach alpha was $84 \%$. The scale changed from 10 points in the preoperative period to 17 points in the postoperative period. Conclusions: The Rhinoplasty Outcome Evaluation is a valid and reliable questionnaire to evaluate the results of rhinoplasty
\end{abstract}

Key words: Rhinoplasty, outcome evaluation, questionnaire.

\section{Resumen}

Introducción: El Rhinoplasty Outcome Evaluation es un instrumento específico para la evaluación cosmética y funcional de la nariz desde la perspectiva del paciente; publicado originalmente en inglés, cuenta con 6 ítems pertenecientes a dominios estéticos y funcionales administrados en preguntas tipo Likert de 5 puntos. El puntaje de la escala puede tomar un valor que va de 6 a 30 puntos, donde el puntaje de 6 es la peor satisfacción con la nariz y 30 la mejor imaginable. El objetivo del presente estudio es traducir y validar este instrumento al español para su uso en Chile. Materiales y Métodos: Se utilizaron las guías de validación lin-

*Recibido el 24 de julio de 2012 y aceptado para publicación el 11 de septiembre de 2012.

Los autores no refieren conflictos de interés.

Correspondencia: Dr. Stefan Danilla E.

Santos Dumont 999, Santiago, Chile

drstefandanilla@gmail.com 
güística del MAPI/TRUST Research Institute. El proceso de validación requirió la traducción inglés-español, contra traducción español-inglés, conciliación inglés-inglés y aplicación de la escala en 5 pacientes. La estabilidad interna se evaluó con el alfa de Cronbach, se utilizó estadística descriptiva y analítica para analizar los resultados. Resultados: Las 5 pacientes evaluadas tuvieron una edad promedio de $22 \pm 4$ años, IMC $23 \pm 4 \mathrm{~kg} / \mathrm{m}^{2}$ y $15 \pm 2$ años de estudio, todas de sexo femenino. La estabilidad interna de la escala fue de $84 \%$ (alfa de Cronbach). Se logró evidenciar que los resultados obtenidos entre el preoperatorio y el postoperatorio de las pacientes, variaron de 10 a 17 puntos, con una mediana de 14 puntos. Conclusiones: El instrumento Rhinoplasty Outcomes Evaluation es un instrumento válido, confiable y reproducible para la evaluación de los resultados de la rinoplastía, desde la perspectiva de los pacientes chilenos hispanoparlantes.

Palabras clave: Rinoplastía, Rhinoplasty Outcomes Evaluation Instrument.

\section{Introducción}

La rinoplastía es una de las cirugías más frecuentemente realizadas por cirujanos plásticos y otorrinolaringólogos a lo largo de todo el mundo; con más de 255.000 procedimientos realizados por año sólo en los Estados Unidos de Norteamérica ${ }^{1}$. Tanto para hombres como mujeres, de distintos grupos etarios y ya sea por motivos estéticos o funcionales; el poder entregar un resultado que cumpla con las expectativas del paciente representa un eterno desafío para el cirujano ${ }^{2}$; no sólo por la destreza o habilidades quirúrgicas necesarias, si no porque la visión del médico tratante puede no coincidir con la del paciente; la "ideación" de la nariz perfecta obtenida por algún estereotipo o fotografía, asociada al gran componente emocional frente a una cirugía estética electiva, hacen difícil evaluar en forma objetiva los resultados de la intervención ${ }^{3,4}$.

En el año 2008, Meningeaud y colaboradores crearon y publicaron el Rhinoplasty Outcomes Evaluation instrument ${ }^{3}$, estudio en el que se aplicó una encuesta de seis ítems donde se logra cuantificar el grado de satisfacción de los pacientes con la apariencia estética y grado de funcionalidad de su nariz, aplicado en el pre y postoperatorio de los pacientes que fueron sometidos a una rinoplastía abierta. Los resultados obtenidos por los creadores han sido replicados por otros cirujanos, con una adecuada consistencia interna y validez de constructo similar al estudio original ${ }^{4}$. Por lo tanto, decidimos emplear el instrumento en nuestros pacientes y evaluar si obteníamos resultados reproducibles, logrando objetivar con un puntaje el nivel de agrado o desagrado frente a los resultados de una rinoplastía. Sin embargo, para la correcta aplicación de un instrumento realizado originalmente en otro idioma, éste debe ser inicialmente traducido y "validado" en el idioma local, de forma que mantenga el mismo grado de consistencia interna y conservar el rigor científico en su aplicación.

El objetivo del presente estudio fue traducir y validar el Rhinoplasty Outcomes Evaluation instrument al español chileno.

\section{Material y Método}

\section{Diseño}

Para lograr nuestro objetivo realizamos un estudio de validación lingüística y adaptación cultural, basados en las guías de validación lingüística MAPI/ TRUST Research institute ${ }^{5}$.

El proceso de validación consta de los siguientes pasos: traducción del instrumento de inglés a español, contra-traducción español a inglés, conciliación inglés-inglés y aplicación de la encuesta en 5 individuos, algoritmo que se muestra en la Figura 1.

\section{Instrumento de medición}

La encuesta publicada en inglés ${ }^{2}$ incluye 6 ítems pertenecientes a dominios estéticos y funcionales de la nariz; administrados en preguntas tipo Likert de 5 puntos (Tabla 1 ).

Las propiedades psicométricas de la escala en su publicación original fueron: Test re test $\mathrm{r}=0,83$, $\mathrm{p}<0,001$; consistencia interna medida con el $\alpha$ de Cronbach $=0,84$; validez medida a través de la "Sensibilidad al Cambio" mediante el puntaje obtenido entre pre y el postoperatorio (paired t test $\mathrm{p}<0,001)^{3,5}$.

La escala tipo "Likert" o evaluación sumaria, es un tipo de escala psicométrica, en la que se especifica el nivel de acuerdo o desacuerdo con una declaración (elemento, ítem, reactivo o pregunta) ${ }^{6}$.

\section{Estadística}

Los resultados se presentan resumidos con estadística descriptiva utilizando promedio \pm desviación estándar para las variables continuas, mediana para las ordinales y frecuencia y porcentaje para las categóricas. La estabilidad interna de la escala se midió con el alfa de Cronbach. La comparación antes-después del puntaje de la escala, se realizó con el test de Wilcoxon para muestras pareadas considerando significativo un valor alfa de 0,0500 (Stata $10.2^{\circledR}$ ).

\section{Resultados}

Luego de la traducción, contra-traducción y conciliación, se contó con un instrumento de 6 ítems, el que se presenta en la Tabla 2. 


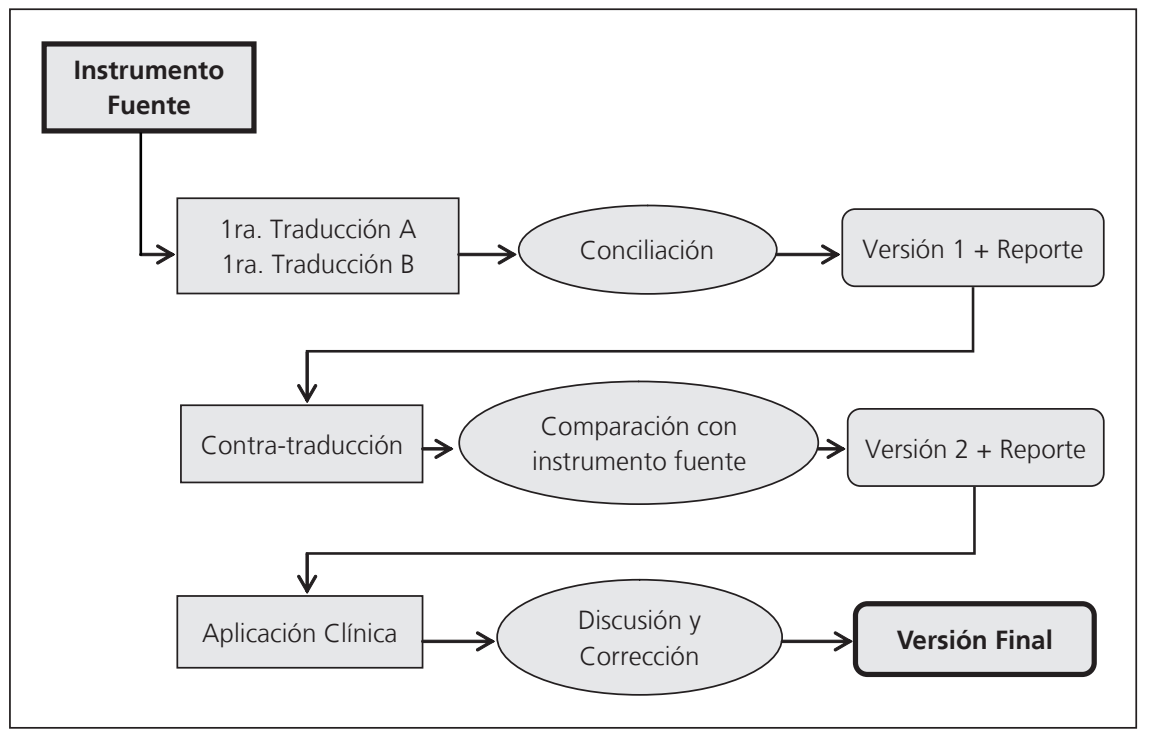

Figura 1. Algoritmo del proceso de validación Lingüística según la MAPI/ TRUST Research institute.

Tabla 1. Encuesta para evaluación de resultados en rinoplastía: Rhinoplasty Outcomes Evaluation instrument

\section{Rhinoplasthy Results Evaluation}

1. How much you like the appearance of your nose?

2. How well you can breathe through your nose?

3. How much you think your friends and loved ones like your nose?

4. Do you think the actual appearance of your nose has limited your social activities or work?

5. How sure are you about your actual appearance of your nose, is it the best you can have?

6. Would you surgically change the appearance and functionality of your nose?
Las características de los pacientes a los cuales fue aplicado el instrumento se presentan en la Tabla 3. Se utilizó una muestra de 5 pacientes que serían sometidos a una rinoplastía abierta en el Hospital Clínico de la Universidad de Chile, por miembros del equipo de Cirugía Plástica; evaluando la validez del instrumento con la misma encuesta tanto en el preoperatorio como en el postoperatorio.

La estabilidad interna fue de $84 \%$.

Las pacientes encuestadas tuvieron una edad de $22 \pm 4$ años, IMC $23 \pm 4 \mathrm{~kg} / \mathrm{m}^{2}$ y $15 \pm 2$ años de estudio, todas de sexo femenino (Tabla 3 ).

En la Tabla 4 se puede observar la mediana de puntaje por ítem en el pre y postoperatorio. Se destaca que en todos los ítems hubo mejoría, con

Tabla 2. Encuesta final traducida, instrumento aplicado a la muestra de 5 pacientes

\begin{tabular}{|c|c|c|c|c|c|}
\hline & $\begin{array}{l}\text { Totalmente } \\
\text { en } \\
\text { desacuerdo }\end{array}$ & $\begin{array}{c}\text { Parcialmente } \\
\text { en } \\
\text { desacuerdo }\end{array}$ & $\begin{array}{l}\text { Ni acuerdo } \\
\text { ni en } \\
\text { desacuerdo }\end{array}$ & $\begin{array}{l}\text { Parcialmente } \\
\text { de } \\
\text { acuerdo }\end{array}$ & $\begin{array}{l}\text { Totalmente } \\
\text { de } \\
\text { acuerdo }\end{array}$ \\
\hline $\begin{array}{l}\text { a) La apariencia de mi nariz es la mejor que } \\
\text { puedo imaginar }\end{array}$ & & & & & \\
\hline b) Puedo respirar perfectamente por mi nariz & & & & & \\
\hline c) A mis amigos y familia les gusta mi nariz & & & & & \\
\hline $\begin{array}{l}\text { d) Nunca me he sentido limitado en mis } \\
\text { actividades sociales-laborales por la } \\
\text { apariencia de mi nariz }\end{array}$ & & & & & \\
\hline $\begin{array}{l}\text { e) Estoy seguro que la apariencia de mi nariz } \\
\text { es la mejor que puedo tener }\end{array}$ & & & & & \\
\hline $\begin{array}{l}\text { f) No me gustaría cambiar quirúrgicamente } \\
\text { la apariencia y funcionalidad de mi nariz }\end{array}$ & & & & & \\
\hline
\end{tabular}


Tabla 3. Características generales de los pacientes evaluados mediante la encuesta

\begin{tabular}{|cccc|}
\hline $\begin{array}{c}\text { Paciente } \\
\mathbf{n}\end{array}$ & $\begin{array}{c}\text { Edad } \\
\mathbf{A n ̃ o s}\end{array}$ & $\begin{array}{c}\text { IMC } \\
\mathbf{k g} / \mathbf{m}^{\mathbf{2}}\end{array}$ & $\begin{array}{c}\text { Escolaridad } \\
\text { Años }\end{array}$ \\
1 & 26 & 19,5 & 17 \\
2 & 18 & 20,3 & 12 \\
3 & 20 & 28 & 14 \\
4 & 27 & 25 & 17 \\
5 & 20 & 21,8 & 14 \\
Promedio & $22 \pm 4$ años & $23 \pm 4 \mathrm{~kg} / \mathrm{m}^{2}$ & $15 \pm 2$ años \\
\hline
\end{tabular}

Tabla 4. Mediana de respuestas por ítem

\begin{tabular}{|lccc|}
\hline & $\begin{array}{c}\text { Preope- } \\
\text { ratorio }\end{array}$ & $\begin{array}{c}\text { Postope- } \\
\text { ratorio }\end{array}$ & $\begin{array}{c}\text { p } \\
\text { value }\end{array}$ \\
\hline $\begin{array}{l}\text { a) La apariencia de mi nariz es la mejor que puedo } \\
\text { imaginar }\end{array}$ & 1 & 4 & 0,0422 \\
\hline b) Puedo respirar perfectamente por mi nariz & 5 & 5 & 0,7773 \\
\hline c) A mis amigos y familia les gusta mi nariz & 3 & 4 & 0,2185 \\
$\begin{array}{l}\text { d) Nunca me he sentido limitado en mis actividades } \\
\text { sociales-laborales por la apariencia de mi nariz }\end{array}$ & 4 & 4 & 0,1159 \\
\hline $\begin{array}{l}\text { e) Estoy seguro que la apariencia de mi nariz es la } \\
\text { mejor que puedo tener }\end{array}$ & 1 & 4 & 0,0339 \\
\hline $\begin{array}{l}\text { f) No me gustaría cambiar quirúrgicamente la apa- } \\
\text { riencia y funcionalidad de mi nariz }\end{array}$ & 1 & 4 & 0,0394 \\
\hline
\end{tabular}

excepción del punto referente a funcionalidad de la nariz y en la limitación social-laboral relacionada a la apariencia de la nariz. No hubo problemas en la comprensión de las preguntas por ninguno de los pacientes encuestados.

Todos los pacientes evaluados mejoraron su puntaje en el postoperatorio $(\mathrm{p}=0,04)$, incrementando el puntaje entre 10 y 17 puntos, con una mediana de 14 puntos $(\mathrm{p}=0,04)$ (Tabla 5).

Como ejemplo, se adjunta la evaluación de una paciente del estudio y su fotografía antes-después (Figura 2).

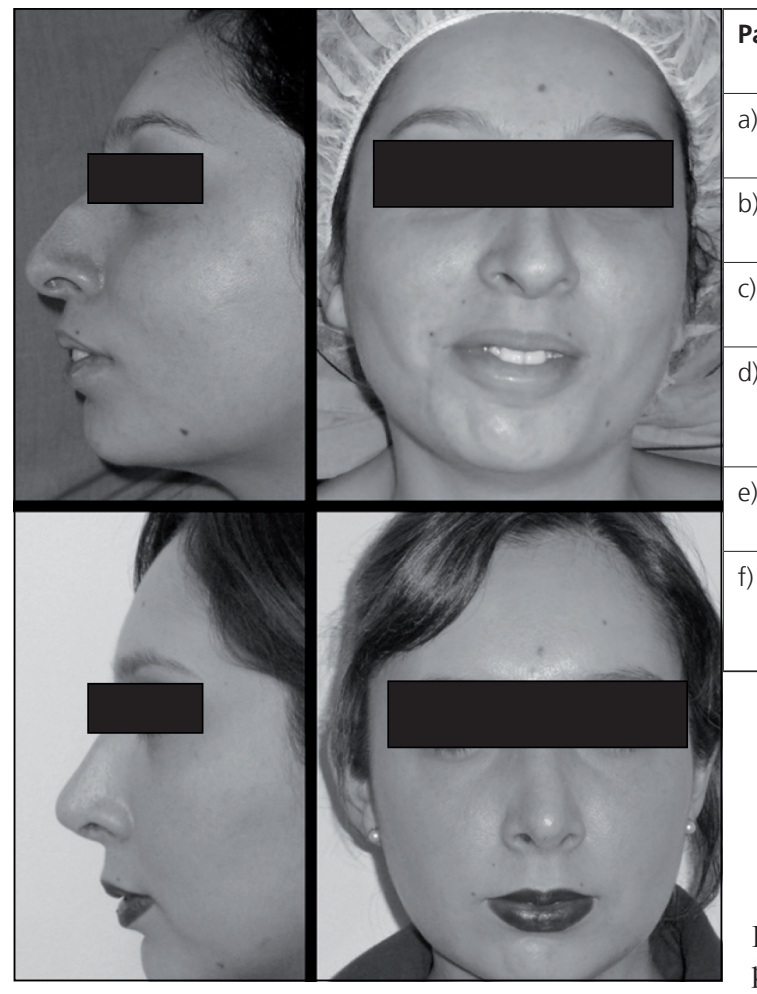

\begin{tabular}{|l|l|c|c|}
\hline \multicolumn{2}{|l|}{ Paciente No } & $\begin{array}{c}\text { Preope- } \\
\text { ratorio }\end{array}$ & $\begin{array}{c}\text { Postope- } \\
\text { ratorio }\end{array}$ \\
\hline a) & $\begin{array}{l}\text { La apariencia de mi nariz es la mejor } \\
\text { que puedo imaginar }\end{array}$ & 1 & 4 \\
\hline b) & $\begin{array}{l}\text { Puedo respirar perfectamente por mi } \\
\text { nariz }\end{array}$ & 2 & 4 \\
\hline c) & $\begin{array}{l}\text { A mis amigos y familia les gusta mi } \\
\text { nariz }\end{array}$ & 2 & 5 \\
\hline d) & $\begin{array}{l}\text { Nunca me he sentido limitado en mis } \\
\text { actividades sociales-laborales por la } \\
\text { apariencia de mi nariz }\end{array}$ & 1 & 5 \\
\hline e) & $\begin{array}{l}\text { Estoy seguro que la apariencia de mi } \\
\text { nariz es la mejor que puedo tener }\end{array}$ & 1 & 4 \\
\hline f) & $\begin{array}{l}\text { No me gustaría cambiar quirúrgica- } \\
\text { mente la apariencia y funcionalidad } \\
\text { de mi nariz }\end{array}$ & 1 & 4 \\
\hline
\end{tabular}




\section{Conclusión}

El poder evaluar el grado de satisfacción de una cirugía por parte del paciente está adquiriendo cada vez una mayor trascendencia ${ }^{7,8}$, tanto del punto de vista científico al permitir objetivar y cuantificar los resultados con una u otra técnica quirúrgica, evaluar una curva de aprendizaje o estudios epidemioló$\operatorname{gicos}^{9-11}$; como también desde el punto de vista de la relación médico-paciente, al poder demostrarle los cambios y hacerlo a él partícipe de su propia mejoría $^{12,13}$.

En la cirugía estética, la disconformidad del paciente con los resultados de la operación, también puede ser considerada como una complicación postoperatoria ${ }^{14}$. El "cuantificar" los cambios obtenidos entre el pre y el posoperatorio nos puede ayudar a disminuir esa sensación en nuestros pacientes.

La versión en Español del Rhinoplasty Outcomes Evaluation nos demostró que es un instrumento válido, confiable y reproducible para la evaluación de los resultados de la rinoplastía, desde la perspectiva de los pacientes chilenos.

La traducción y validación de la encuesta ROE en "español-chileno" fue realizada para su aplicación a nivel local; sin embargo la "neutralidad" de la traducción, permite su aplicación a nivel de cualquier grupo de pacientes hispanoparlantes.

\section{Referencias}

1. American Society of Plastic Surgeons. National Clearinghouse of Plastic Surgery Statistics. http://plasticsurgery.org/Documents/Media/statistics/2009- US - cosmetic reconstructive plastic surgery minimally-invasive statistics. Pdf Sept 2010;1.

2. Luce E. Outcome studies and practice guidelines in plastic surgery. Plastic and Reconstructive Surgery 1999; 104:1187-90.

3. Meningaud JP, Lantieri L, Bertrand JC. Rhinoplasty: An
Outcome Research. Plastic and Reconstructive Surgery. 2008;121:251-7.

4. Alsarraf L. Outcomes research in facial plastic surgery: A review and new directions. Aesthetic Plast. Surg. 2000;24:192-7.

5. CMapi Research Institute. Linguistic Validation of a Patient Reported Outcomes Measure. Lyon-France. 2005.

6. Likert R,"A Technique for the Measurement of Attitudes". Archives of Psychology 1932;140:44-53.

7. Kazis L, Anderson J, Meenan R. Effect sizes for interpreting changes in health status. Med Care 1989;27:178S189.

8. Ramsey A, Larrabee W, Anderson S, Craig M, Calvin M. Measuring Cosmetic Facial Plastic Surgery Outcomes. Arch Facial Plast Surg. 2001;3:198-201.

9. Cano S, Klassen A, Pusic A. The science behind qualityof-life measurement: A primer for plastic surgeons. Plastic and Reconstructive Surg. 2009;123:98-106.

10. Fitzpatrick R, Jenkinson C, Klassen A, Goodacre T. Methods of assessing health-related quality of life and outcome for plastic surgery. Br J Plast Surg. 1999;52:251-5.

11. Cano S, Browne J, Lamping D. Patient-based measures of outcome in plastic surgery: Current approaches and future directions. Br J Plast Surg. 2004;57:1-11.

12. Thoma A, Veltri K, Khuthaila D, Rockwell G, Duku E. Comparison of the deep inferior epigastric perforator flap and free transverse rectus abdominis myocutaneous flap in postmastectomy reconstruction: A costeffectiveness analysis. Plastic and Reconstructive Surg. 2004; 113:1650-61.

13. Efficace F, Bottomley A, Osoba D, Gotay C, Flechtner $\mathrm{H}$, D'haese S, et al. Beyond the development of healthrelated quality-of-life (HRQOL) measures: A checklist for evaluating HRQOL outcomes in cancer clinical trials. Does HRQOL evaluation in prostate cancer research inform clinical decision making? J Clin Oncol. 2003;21:3502-11.

14. Carr-Hill R. The measurement of patient satisfaction. J Public Health Med. 1992;14:236-49. 